\title{
VISUALISATION OF LIQUID FLOW PHENOMENA IN TEXTILES APPLIED AS A WOUND DRESSING
}

\author{
A. Komisarczyk', G. Dziworska', I. Krucinska', M. Michalak', W. Strzembosz', A. Kaflak², M. Kaluza ${ }^{3}$ \\ 'Lodz University of Technology, Department of Commodity and Material Sciences and Textile Metrology, Center Advanced Technologies of Human Friendly Textiles \\ ${ }^{2}$ Medical University of Warsaw, Faculty of Pharmacy, Department of Inorganic and Analytical Chemistry \\ ${ }^{3}$ Lodz University of Technology, Institute of Electronics \\ e-mail: izabella.krucinska@p.lodz.pl
}

\section{Abstract:}

The aim of this work was to visualise liquid transport in textiles. Knowledge of the transport phenomena allows for the design of textiles for various applications, e.g., comfortable to wear filtration and wound dressing. To visualise liquid transport through textiles, three test methods were explored. The first one was the high spatial resolution magnetic resonance imaging (MRI) technique (also referred to as nuclear magnetic resonance (NMR) microscopy). It allowed the observation of the pathways of liquid flow through textiles. In the second method, a thermographic camera was used to record temperature changes and assess the liquid flow in the textile. The third method was using a high-speed video camera to observe the liquid transport within the textile. Two types of textiles were studied: a double-layer knitted fabric and a woven fabric, both made from hydrophilic and hydrophobic fibres (cotton, viscose and polypropylene). The knitted fabrics were tested as a new type of wound dressing, which transported moisture with adequate control; therefore, it could be used as a substrate for a drug delivery system.

\section{Keywords:}

Mass transport, physiological comfort, fluid flow, wound dressings

\section{Introduction}

The transport of liquids is very important for the design, applications and functionality of textiles. Wetting processes are considered extremely important in fibrous medical textiles applications, where wetting of the fibre surface is the key mechanism for the healing process and for the transport of liquid drugs into the wound. The wetting and wicking behaviour of the fibrous structure is a critical aspect of performance in products, such as disposable hygiene materials and medical items [1]. The above-mentioned phenomena are very complex processes that could be made difficult by the structure of the fibrous construction. While the transport phenomena depend on the fabrics structure, i.e., their pattern and finishing, the structure of the fibrous construction depends on the curvature of the fibres, crimps on the fibres and fibre orientation. The surface of the textile has also a strong influence on water transport. Using a nanofibre web made of hydrophobic materials one can create, for example, superhydrophobic surfaces whereas electrospunnanofibrous webs can be used in protective systems as barriers to liquid penetration. The phenomenon of liquid transport through textiles should therefore be regarded as a very important process.

The moisture transport through textile products has been studied experimentally and theoretically. Nilgün ÖZDiL et al. [2] have studied the moisture management of cotton knitted fabrics in the single jersey structure. The properties were measured using a SDL-ATLAS moisture management tester. The authors measured the dynamic liquid transport properties, such as wetting time, maximum absorption rate and spreading speed of the liquid in textiles. It was found out that the yarn count and yarn twist coefficient had a significant influence on these properties. Kristian Krabbenhøft [3] studied physicomathematical models of moisture transport in wood and their numerical implementation. In the afore-mentioned study, the structure and basic features of wood, related to moisture transport, were briefly discussed, followed by a review of the general theory of moisture transport in porous media. Relevant conservation equations were provided, and the constitutive relations governing the transport of the different water phases were discussed, i.e., the problems of moisture transport below the saturation point, of free water flow above the saturation point and of wood preservation. The problems of drying were also discussed, and a three dimensional wood drying example was presented. The author presented also the numerical methods for the solution of the proposed models. In the article by Guowen Song [4], the author presented a numerical model of heat and moisture transport in thermal protective clothing during exposure to a flash fire. The model was developed with the assumption that textiles are porous media. Roger $L$. et al. [5] developed a model of heat and moisture transport in fire fighters protective clothing during a flash fire exposure. A traditional fire fighter garment consists of three layers of fabric (the outer shell, the moisture barrier, and the thermal liner) that transport heat and moisture to the human skin and tissue. Researchers traditionally focused on studying the heat transfer phenomenon in fabrics during flash fire exposures. Moisture transport and its impact on the performance of protective garment have not been studied in sufficient detail. Therefore, the knowledge of the distribution of liquid in the structure of fabrics is required. One way to increase the understanding of the process is to visualise it. Liquid distribution in textiles could be visualised using techniques, such as magnetic resonance imaging (MRI), thermal imaging, and high-speed video camera imaging. 
Mass transport characteristics within textiles are extremely important. Textile structures having a good moisture transport characteristics are used, among others, as wound dressings and different multifunctional textiles have been developed in the last few decades for such purposes. Wound dressings must present exceptional biocompatibility and remain in good biophysical condition for tissue regeneration. According to numerous studies, wounds should be kept wet, and the material that covers destroyed tissue should be removed from the skin with an overflow of exudation [7]. Moreover, only some particles, such as peptides, bacteria and dead cells, should be removed from the wound. Elements of exudation, such as water, mineral salts, ions and some enzymes, should stay in the wound. Thus, the porosity and pore distribution of wound dressings are also important.

Many wound dressings are used not only as wound covers, but also as part of a drug delivery system. In such case a predetermined amount of drugs needs to be delivered over a required time period. Thus, new and intelligent drug delivery systems are being studied. Those systems are based on the microcapsulation technology, where the shell of the microcapsules is degraded by the conditions present in the infected wound, such as specific $\mathrm{pH}$ values or characteristic groups of enzymes [8]. The drug delivered to the wound is released in a flow opposite to the exudation. Thus, most of the drug released from microcapsules in typical sorptive textiles would be transported by exudation to the sorption layer and would become inactive [8].

\section{Experimental results}

The work presented in this section was divided in two parts. First, the technology of a new type of textile for wound dressings was developed. The main goal of the study was creation of the specific structure of a fabric with high porosity and an appropriate pore diameter, enabling the transport of moisture. The structure of the textile should have two types of "channels", enabling the transport of moisture and the transport to the wound of the drug released from the microcapsules, using different pathways.

The textiles were also assumed to have high sorption capacity and low sorption rate, which would ensure that exudation overflow would be collected, whereas the wound would remain wet. It is well known that strongly hydrophilic materials are characterised by a high sorption rate and that hydrophobic materials have a low sorption rate. Moisture could be retained by hydrophilic fibres, whereas moisture could be transported using hydrophobic threads. Thus, the material should consist of two layers: the hydrophobic layer being the transport pathway and the hydrophilic layer enabling moisture accumulation.

All biophysical properties of the selected textiles were characterised. The main parameters of the structure, such as surface mass, thickness, apparent density and pore diameter were also measured. The properties were examined according to the European Standards. The measurements of the surface mass were made according to EN-29073-1:1994. The measurements of the thickness were made according to EN 5084:1999. The air permeability of the fabrics was assessed according to EN ISO 9237:1998, and the moisture vapour transmission rate (MVTR) of the permeable film dressing was done according to EN 13726$2: 2005$. Finally, the internal pore diameter was calculated based on the air permeability, using the following equation:

$$
d_{k}=\sqrt{\frac{256 R T \eta g \cdot \dot{m}}{\pi D^{2}(2 P-\Delta p) \varepsilon \Delta p}}
$$

where: $\mathrm{j}$ - number of channels - -, D - flow diameter - m, gthickness of textile fabric- $m, \varepsilon$ - porosity - - , $d_{k}-$ channel diameter - $\mathrm{m}, \mathrm{T}$ - air temperature - $\mathrm{K}, \Delta \mathrm{p}$ - aerodynamic resistance of fabric - $\mathrm{Pa}, \mathrm{R}$ - gas constant - $\mathrm{Jmol}^{-1} \mathrm{~K}^{-1}, \mathrm{~h}$ - air dynamic viscosity - Pa s.

The second part of the research concentrated on the visualisation of the liquid transport in textiles. The moisture distribution in the textile structures was imaged using MRI, by recording the liquid flow within the textiles using a high-speed video camera, and by measuring the temperature changes within the textiles with a thermographic camera.

\subsection{Tested material}

Two techniques were used to create the multilayer textile: knitting and weaving. They allowed to produce materials with good sorption and mechanical properties, even when wet. To ensure biocompatibility within the final multilayer textile, two types of threads (cellulosic and polypropylene) were used to create two variants of knitted fabric. The first one, marked KF1, was made from polypropylene (PP) and cotton (CO) threads. The second one, marked KF2, was made from PP and viscose (Vi) threads. Knitted fabrics were produced using a rib-knitting machine.

Hydrophobic layers made of PP threads created channels that crossed the boundary between layers. The channel structure facilitated moisture transport from the wound to the hydrophilic layer. The mechanism of moisture and drug flow in the knitted fabric structure is shown in Figure 1.

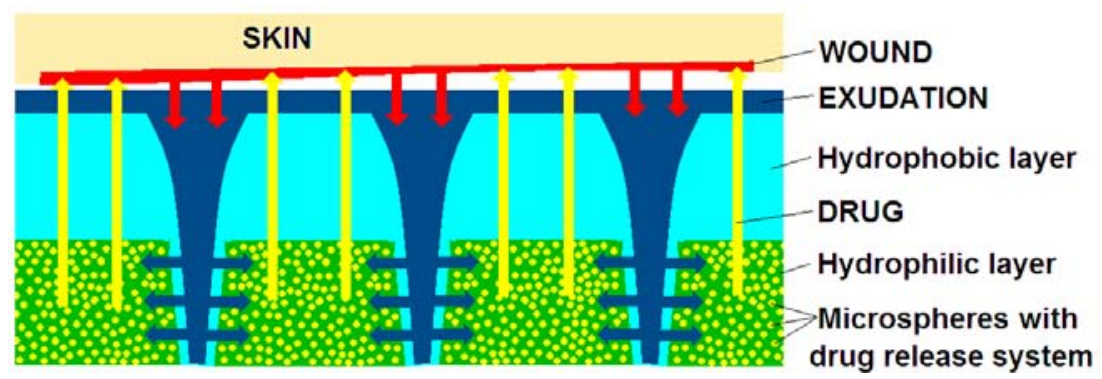

Figure 1. The mechanism of moisture and drug flow in the knitted fabric structure. 
Wound exudation is transported by hydrophobic channels to the hydrophilic layer made of cotton or viscose, where microspheres with the drug release system are located. Enzymes from the exudation are degrading microspheres shells, thus releasing the drugs. Drugs are then transported to the wound in a direction opposite to the direction of moisture flow.

Pictures of the fabrics are presented in Figure 2. Figure 3 shows the micrographs of the cross section and the sectional view of the knitted fabrics.

The pathway of liquid flow through the textiles is clearly visible on the Figure 3. However, the phenomena of liquid flow through the textiles is more complicated, because of the liquid migration process, which could influence the fibre structure.
The properties of the knitted fabrics were evaluated and are shown in Table 1.

During the research, an additional type of textile structure was designed: a layered and thinly woven fabric made from PP (hydrophobic) and cellulose LCY (hydrophilic) fibres. The fabric was produced using satin weave and its properties were evaluated using the same procedures as before. The structure of the woven fabric is presented in Figure 4 . In Figure 5 and 6 are presented the micrographs of the cross section and the sectional view of the knitted fabric. The measured parameters and properties of the woven fabric are summarized in Table 1.

In the figures above (1) is the PP thread, and (2) is the lyocell thread.
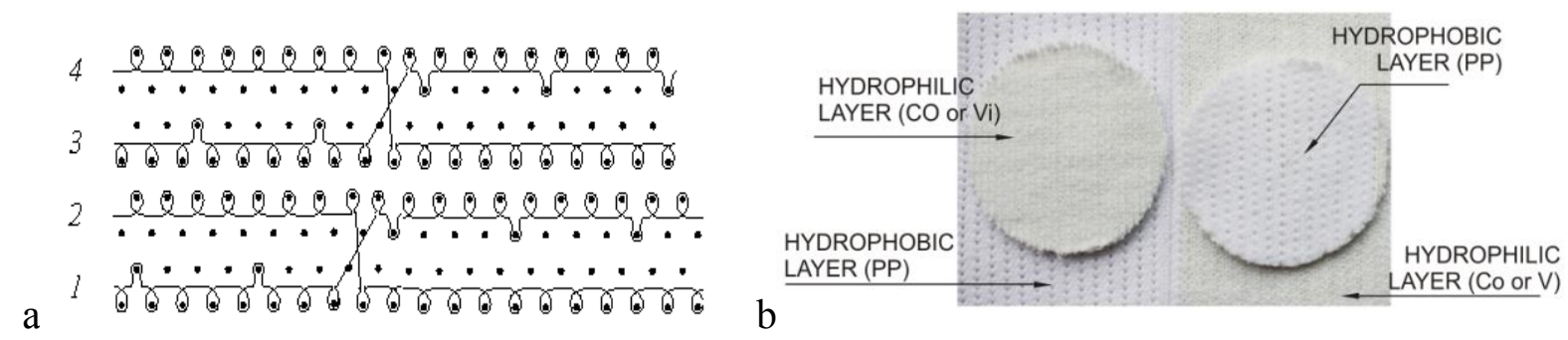

Figure 2. Structure of the knitted fabric made from hydrophobic (PP) / hydrophilic (cotton or viscose) layers. a) The threads scheme (stitch) in knitting wound dressing KF -1 and KF 2. b) Some channels are visible on the hydrophobic layer.

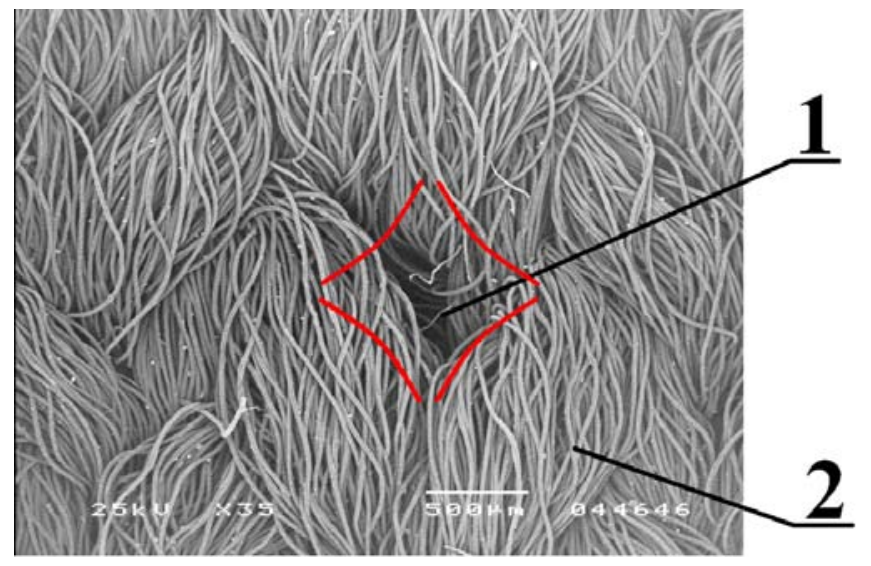

Figure 3. Surface of the knitted fabric KF1, with one visible channel (1) and multifilament PP (2).

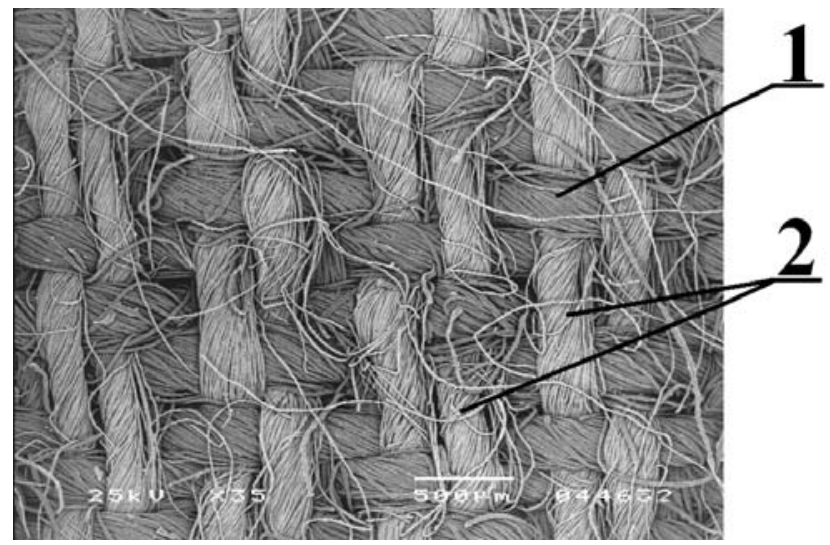

Figure 5. Sectional view of woven fabrics.and multifilament PP (2).

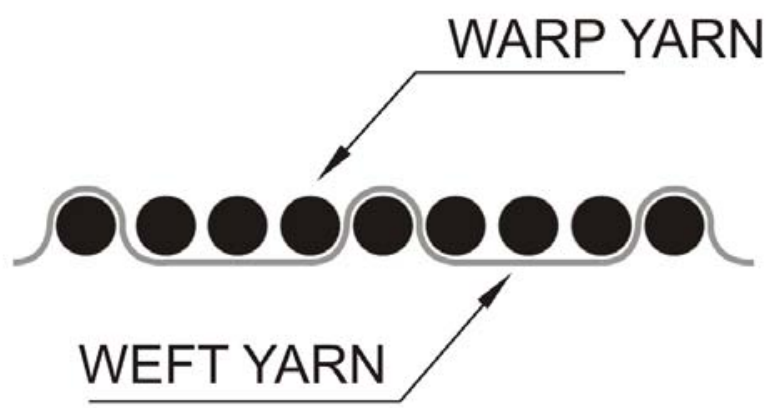

Figure 4. Scheme of weave of woven fabrics.

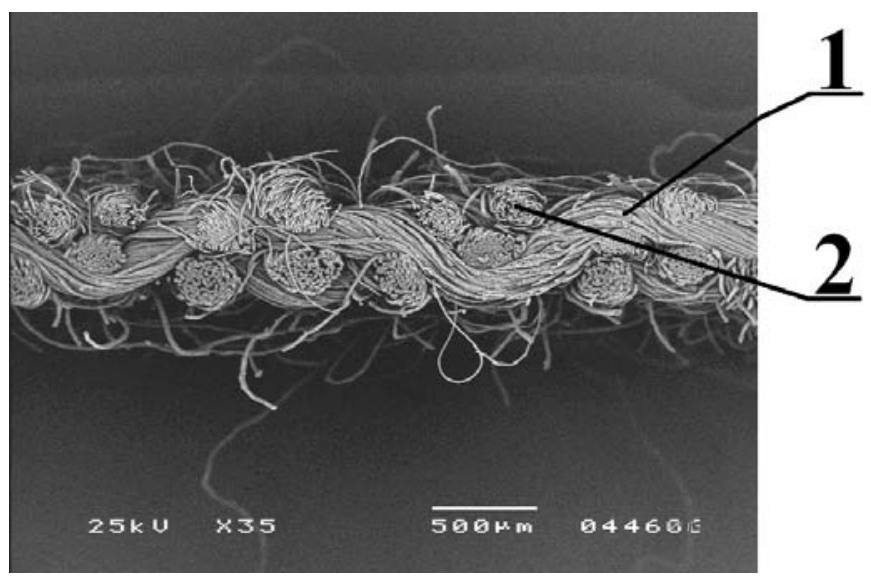

Figure 6. Cross section of woven fabrics. 
Table 1. Properties of the knitted and woven fabrics.

\begin{tabular}{|c|c|c|c|c|}
\hline Sample name & & $\mathrm{KF}^{*}$ & $\mathrm{KF}^{*}$ & PP/LCY \\
\hline Thickness & $\mathrm{mm}$ & 2.63 & 2.40 & 0.68 \\
\hline Surface mass & $\mathrm{g} / \mathrm{m}^{2}$ & 408.00 & 574.90 & 205.92 \\
\hline Coeff. of variation of mass & $\%$ & 0.43 & 1.53 & 1.29 \\
\hline Apparent density & $\mathrm{kg} / \mathrm{m}^{3}$ & 152.24 & 239.55 & 303.71 \\
\hline Air permeability at 2 Pa & $\mathrm{dm}^{3} / \mathrm{m}^{2} \mathrm{~s}$ & 64.5 & 91.4 & 110.1 \\
\hline Air permeability at 5 Pa & $\mathrm{dm}^{3} / \mathrm{m}^{2} \mathrm{~s}$ & 128.3 & 194.2 & 217.0 \\
\hline Internal pore diameter & $\mathrm{m}$ & 0.000252 & 0.000274 & 0.000165 \\
\hline Moisture vapour transmission rate & $\mathrm{gm}^{-2} 24 \mathrm{~h}^{-1}$ & 4884.8 & 4734.9 & 6295.81 \\
\hline Raw material & $1 \mathrm{st} \mathrm{layer}$ & PP & PP & PP \\
\cline { 2 - 5 } & 2nd layer & Cotton & Vicose & LCY \\
\hline
\end{tabular}

\subsection{Visualisation method}

\subsubsection{NMR/MRI method}

The high spatial resolution NMR/MRI technique was chosen for the purpose of the research presented in this article, because it is suitable for measuring liquid distribution and other relevant properties of liquids entrapped in textiles. The tested samples were invisible in NMR, however, the water molecules not bonded with the fibres, e.g., by hydrogen bonds, were visible using NMR.

The MRI cell had to be modified and adapted for the fabric samples used during experiments. The method of delivering water to the sample, located in a strong magnetic field, had also to be modified. The test setup is presented in Figure 7 , while its cross section is shown in Figure 8.

A special test sample holder was designed for the purpose of the experiments (Figure 9). The holder, made of PTFE and invisible in NMR spectroscopy, ensured repeatable sample location and prevented uncontrolled liquid flow. As shown in Figure 9, the fabric sample was set up on the punching plate labelled $\mathrm{CC}$. Water was delivered to the fabric sample using the channel labelled AA. Excess water that saturated the fabric sample was collected in the reservoir labelled DD.

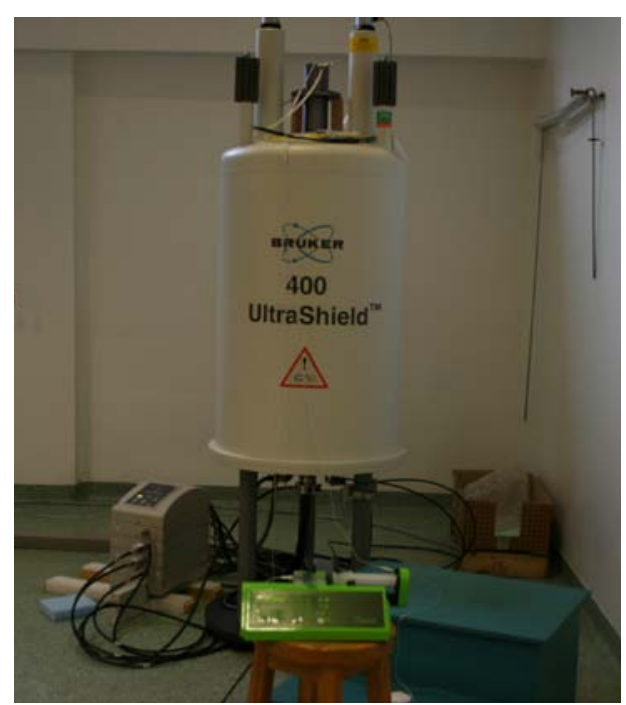

Figure 7. Photo of the NMR test setup.
The experimental system used a vertical NMR magnet. The fabric sample was located within a very strong magnetic field in the MRI detection cell. The holder was set up in the MRI cell and connected to a syringe pump, which supplied water to the samples at a rate of $5 \mathrm{~cm}^{3} / \mathrm{h}$.

\subsubsection{Thermal imaging using the thermographic camera}

Thermal imaging was performed using a MWIR Cedip Titanium camera with a cooled InSb detector matrix $(640 \times 512$ pixels, $15 \mu \mathrm{m}$ pixel pitch) and a noise equivalent temperature difference (NETD) value of $<20 \mathrm{mK}$. The accuracy of the temperature measurement given by the manufacturer was $\pm 1^{\circ} \mathrm{C}$ or $\pm 1 \%$,

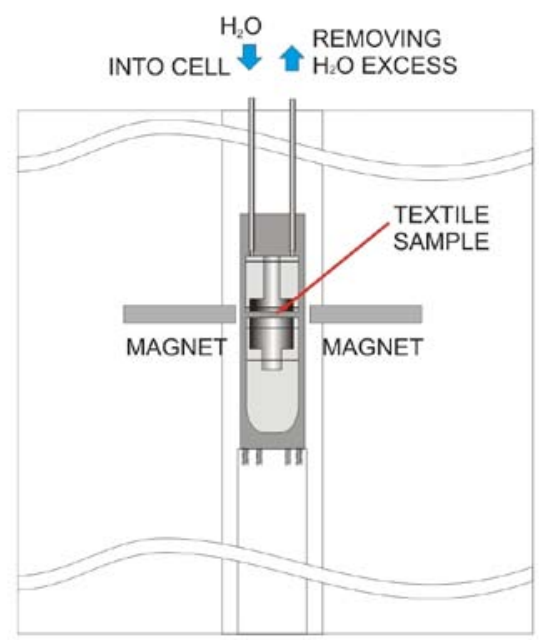

Figure 8. Cross section of the modified NMR setup.

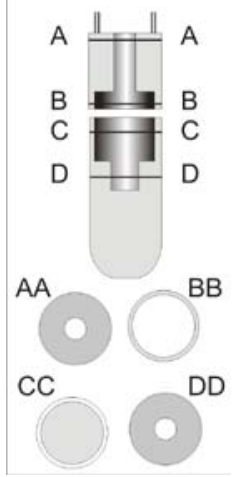

Figure 9. Modified fabric sample holder. 
however, the actual accuracy depended on the type and conditions of the measurements. During measurements, the camera was controlled using a PC computer system with a bidirectional CAM LINK interface and a dedicated Altair program, used to set up camera parameters, acquire data and visualise the results.

To facilitate the measurements, the camera was mounted horizontally on a tripod. To increase the optical resolution of the measurement system, an anodized aluminium extension tube was mounted between the camera housing and the camera lens. The distance from the lens to the nearest edge of the tested fabric sample was $15 \mathrm{~cm}$. The sample was placed in a holder, exactly at the level of the optical axis of the lens.

Five rectangular samples were investigated, prepared from knitted and woven fabrics with different structural parameters. The samples were prepared in such a way, that their edges facing the camera were sharp and parallel to the weft thread or warp thread. All listed materials were tested, but only the results for the woven sample and the knitted fabric KF1 are presented.

Ageneral view of the measurement setup is shown in Figure 10.

\subsubsection{Flow visualisation using a high-speed video camera}

Moisture flow within the textiles was imaged using a Phantom v9.1/3 GB high-speed CCD video camera with a $105 \mathrm{~mm}$ Nikkor macro lens. The recordings were made at $300 \mathrm{fps}$ (equiv. to $3,333.25 \mathrm{~ms}$ period), using a $400 \mathrm{~ms}$ exposure time and 1,200 $\times 600$ pixels resolution. To facilitate the measurements, the camera was mounted horizontally on a tripod. The samples were placed in a holder, exactly at the level of the optical axis of the lens. Rectangular fabric samples were prepared for the tests in such a way, that their edges facing the camera were sharp and parallel to the weft threads and warp threads (in the case of knitting fabrics: wales and courses, respectively), to obtain a clear view of moisture flow through the cross section of the fabric. A general view of the test setup is shown in Figure 11.

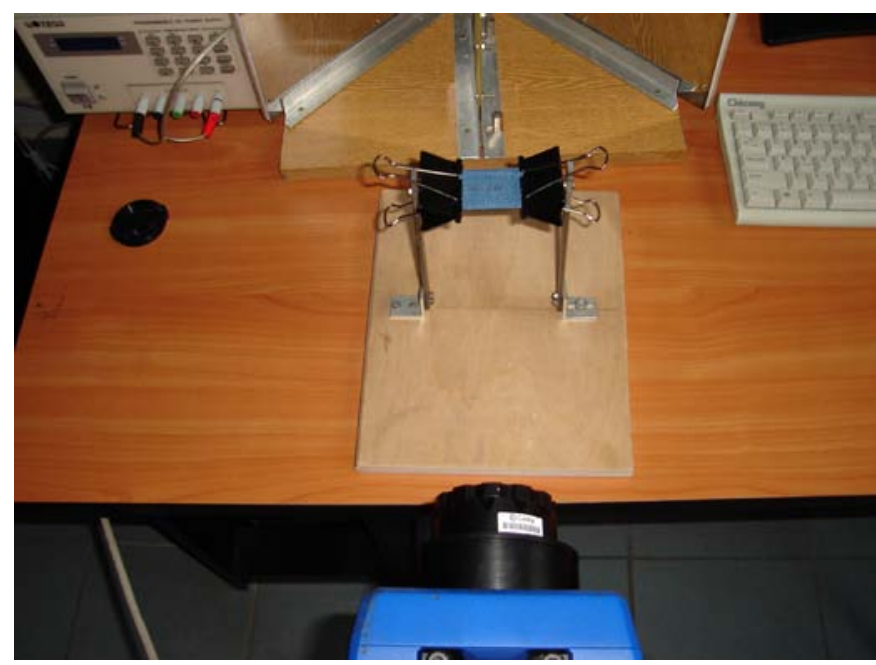

Figure 10. The test setup used to measure moisture flow using Cedip Titanium thermographic camera.

\subsection{Results}

\subsubsection{NMR/MRI method}

In the photos below (Figure 12) are shown the images of liquid flow in textiles, recorded using MRI. The samples themselves are not visible. The white spots visible in the photos are coming from free $\mathrm{H}^{+}$ions present in the water. No signals were recorded when water molecules were chemically bonded to the sample. Water droplets from the water delivery system wetted the hydrophobic side of the material, which simulated the layer contacting the wound. The interval of the photos taken for each sample depended on the sorption rate. The measurement ended when a continuous line of water was visible above the hydrophilic layer. This indicated that the sample was saturated with moisture. The pictures showed visible water molecules inside the hydrophobic layer, under the hydrophobic layer.

In Figure 12a, a visible white line indicates the moisture accumulated between the fibres of the hydrophobic layer. Because no chemical reaction occurred between the water and the polypropylene fibres, free $\mathrm{H}^{+}$ions were present and could be recorded in the NMR images. At this stage, the moisture did not saturate the entire hydrophilic layer. After the hydrophobic layer was saturated, a thin film of water was created between the hydrophilic and hydrophobic layers. It is visible as a bright thin line in Figure 12b. Next, moisture was transported by the fabric channels to the hydrophilic layer. This is visible in Figure $12 b$ as white spots above the bright thin line. After the entire sample was saturated with moisture, the excess water was collected under it. At this stage, the amount of absorbed water was equal to the sorption capacity of the sample. The woven fabric, due to its thickness (less than $1 \mathrm{~mm}$ ) cannot be correctly observed by this method.

\subsubsection{Thermal imaging using a thermographic camera}

Thermography is based on the measurements of the energy radiated by a body having a temperature higher than absolute zero. The radiated energy will depend on the body temperature and on its emissivity coefficient (values in the 0,1 range,

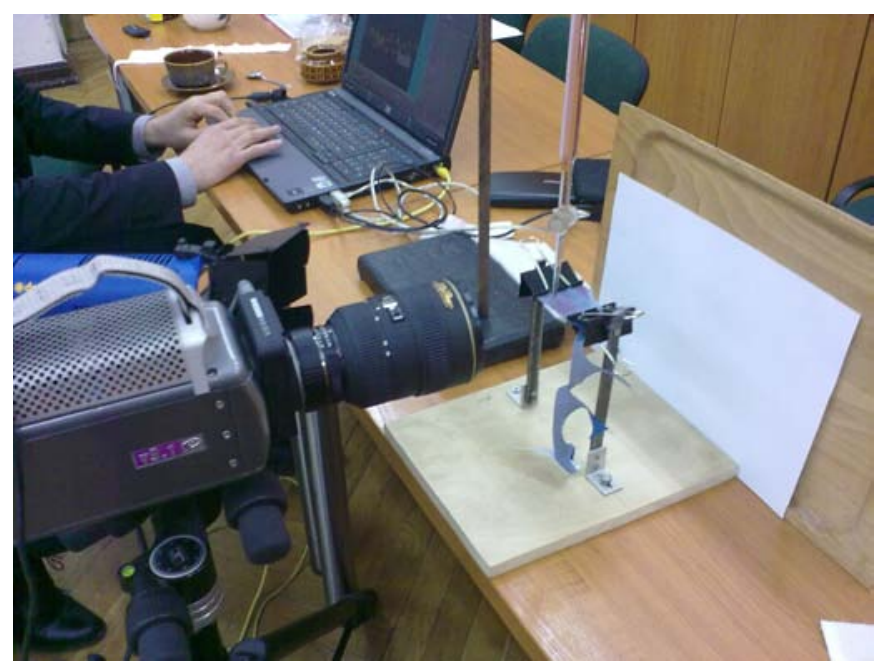

Figure 11. The test setup used to measure moisture flow using a Phantom v9.1/3 GB high-speed video camera. 

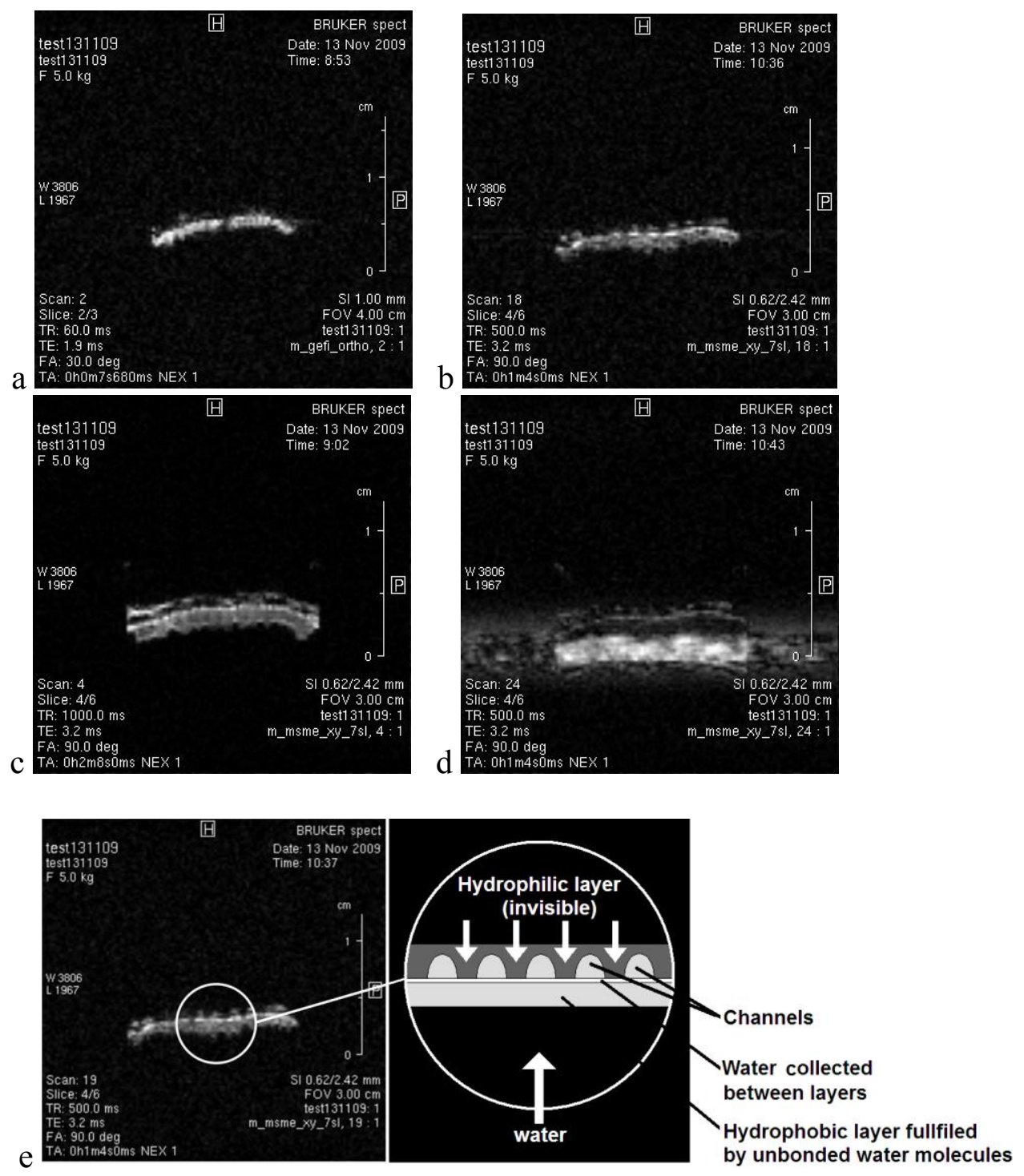

Figure 12. Moisture flow within the knitted fabric. a - the initial state; b - channels in the hydrophilic layer filled with water; $c$ - water-filled hydrophilic layer; $\mathrm{d}$ - overflowed moisture; e - schematic view of the fabric.

around $0.65-0.8$ for most of the fabrics). For two bodies at the same temperature, the one having a higher value of the emissivity coefficient will radiate more energy, and the one having a lower emissivity will radiate less. The emissivity coefficient of the water is lower than the emissivity coefficient of the moistened or unmoistened sample.

To investigate the liquid penetration process in the sample, a pipette was placed $3 \mathrm{~mm}$ away from the sample surface. The thermographic camera began recording the wetting process just before one single drop of water was released on the sample surface. In thermographic images, regions with different temperatures were visible as regions with different colours. The improve the contrast of images, a greyscale palette was used. Thermal images were recorded at 200 frames per second for $30 \mathrm{~s}$ (total of 6000 frames). Only one sample (1-12) was recorded for $60 \mathrm{~s}$.

Because the aim of the paper was to investigate the kinetics of water permeation and not the thermodynamic processes, only the temperature results were analysed, i.e., the temperature on the upper and on the lower surface of sample. To facilitate image analysis, fabric surface markers were used. An example thermogram with markers is shown in Figure 13.

Using the camera software, the temperature at point 1 (blue marker) and at point 2 (green marker) was evaluated.

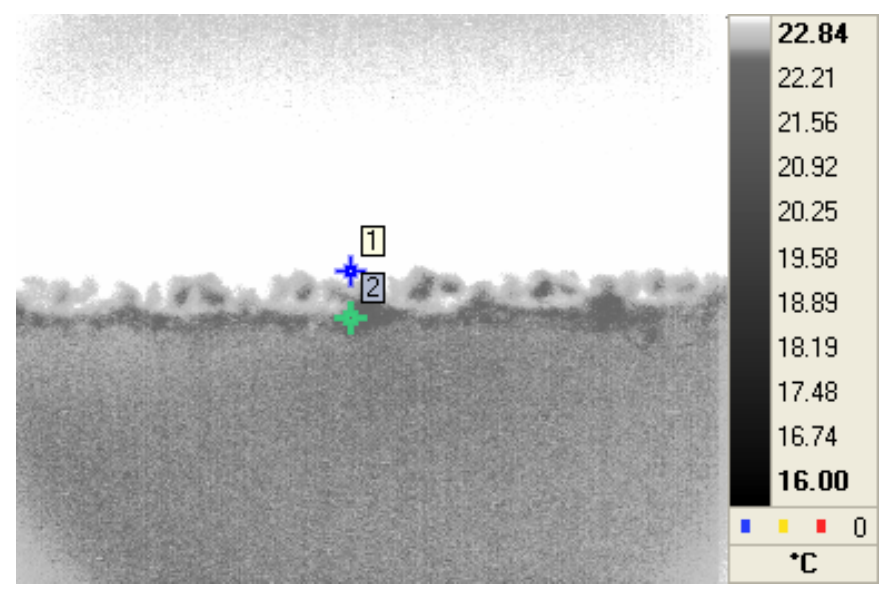

Figure 13. An example thermogram of the woven fabric with markers. 
Based on the temperature and the time estimated from frame numbers, it was possible to analyse how the water penetrated the surface of the sample and flowed to the other surface. For the first presented case (woven fabric), the drop of water contacted the surface at frame 1,203 , which corresponds to $6.015 \mathrm{~s}$. At that moment the temperature at point 1 on the upper surface decreased abruptly by $2.5{ }^{\circ} \mathrm{C}$ (from $23.5{ }^{\circ} \mathrm{C}$ to $21^{\circ} \mathrm{C}$ ). In the next frame, the temperature decreased further to $17^{\circ} \mathrm{C}$. The temperature of the opposite surface did not change noticeably, but started to decrease steadily as the water penetrated the fabric. Example thermograms selected from the entire measurement sequence are presented below (Figure 14), to illustrate characteristic moments of the process.

Figure 14a shows the thermograms in the initial stage, when the water drop first contacted the upper surface of sample. Figures $14 \mathrm{~b}$ and $14 \mathrm{c}$ are showing the water penetration, while it saturates the entire sample.
The results of the measurements for the knitted fabric KF - 1 are shown in Figure 15.

When the drop of water contacted the woven fabric at $4.48 \mathrm{~s}$, it did not moisten it for $0.545 \mathrm{~s}$. During the next $0.44 \mathrm{~s}$, its surface was wetted. After $6.52 \mathrm{~s}$, a portion of the water still remained on the upper surface. After $8.02 \mathrm{~s}$, the water drop penetrated the fabric and reached the lower surface. In the case of the knitted fabric tests, thermal images are showing clearly the pathways of liquid transport through the cross section of the sample (Figure 15).

In Figure 15a, the liquid contacted the sample. Next, liquid pathways are created in the cross section of the textile (Figures 15b and 15c). Due to the temperature differences between water, the fabric and the air, the water flow is visible as dark grey lines. Figure $15 \mathrm{~d}$ shows the sample saturated with liquid. The results for second type of knitted fabric KF - 2 were very similar to presented. We do not observed important differences between water flow through the samples KF-1 and KF - 2 .

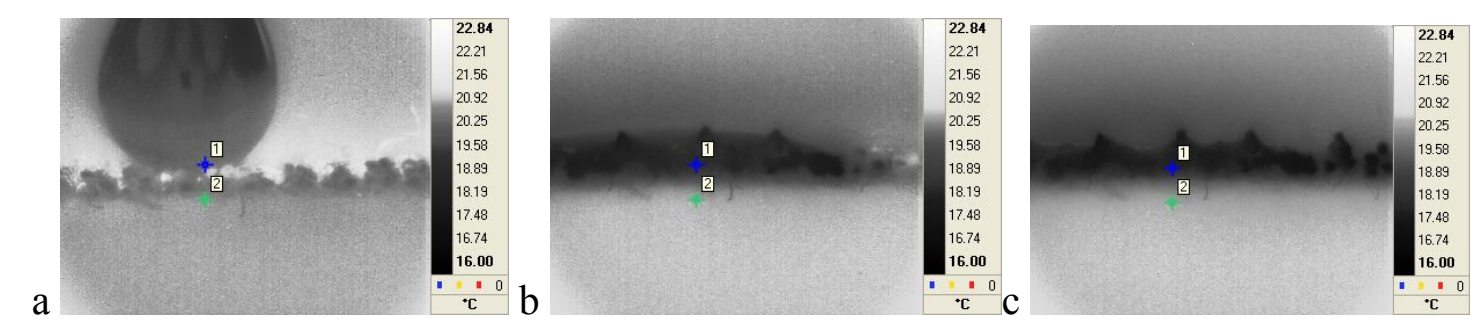

Figure 14. The thermograms of liquid penetration in the woven fabric. a - initial wicking, b, $c-$ creation of channels and flow of the liquid.
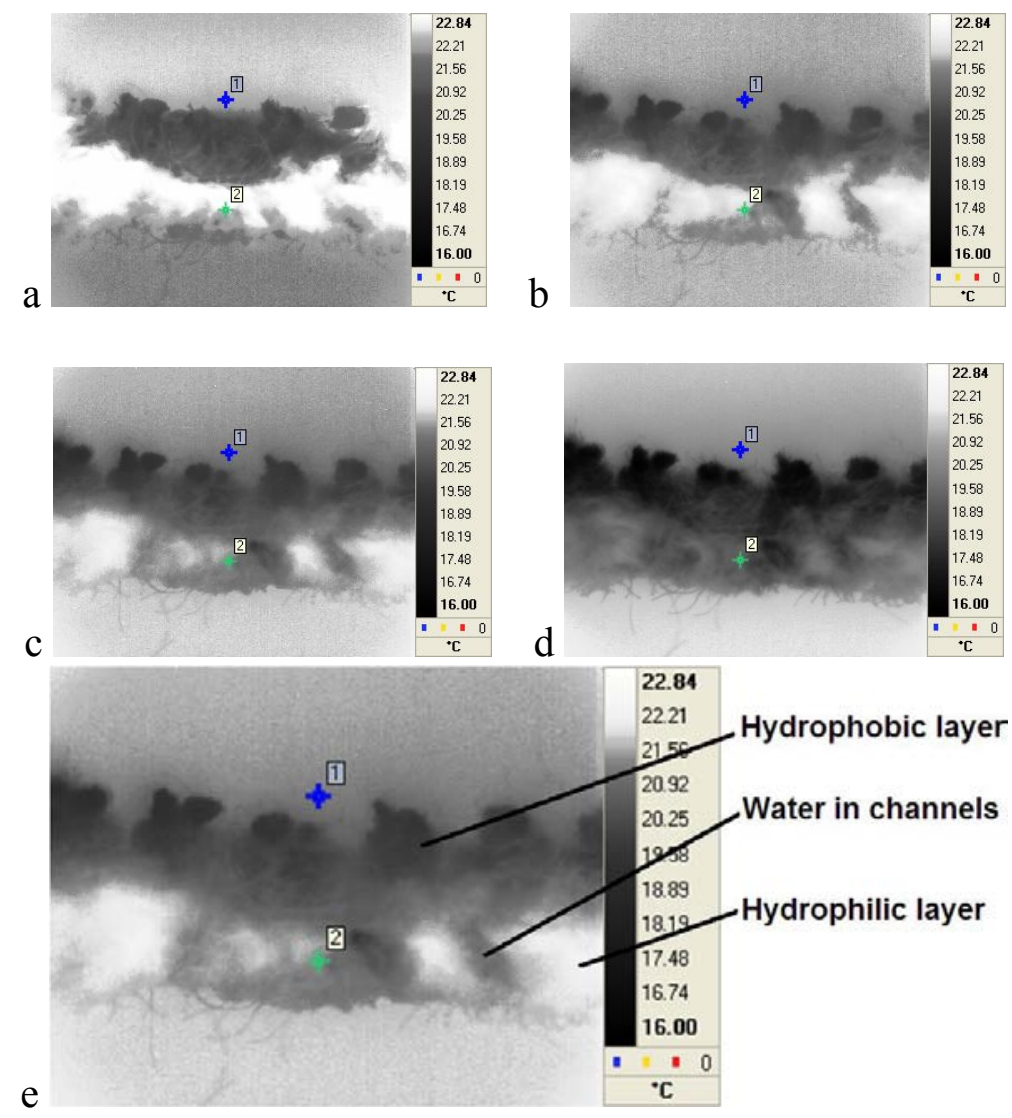

Figure 15. The thermograms of liquid penetration in the knitted fabrics. a - initiation of the flow transport, b-creation of channels, c, $d$ - overflow of water in the sample, $\mathrm{e}-$ interpretation of temperature changes due to water flow. 


\subsubsection{Flow visualisation using a high-speed $C C D$ video camera}

Moisture flow was recorded using a high-speed CCD video camera. As a test liquid water with Congo Red dye was used to increase the visibility of the moisture transport process. The film obtained from the high-speed camera shows that moisture was transported through the cross section of the sample. Representative frames were chosen from the film and analysed using the CineViewer 663 software. They are presented below in Figure 16.

During the initial stage, only a part of the hydrophobic layer was filled. Moisture was collected between the fibres. Then, the existing channels transported the moisture to the hydrophilic layer, where it was accumulated. The moisture flow inside of both knitting fabric were similar, no differences were observed. The same phenomena was also observed in the woven fabrics. In that case, however, the fabric was too thin to allow the recording of moisture transport (Figure 17), and the results could not be interpreted.

The presented results suggest that the possibility of visualising moisture flow through the fabric using a high-speed video camera is limited by the thickness and density of the sample and of course, by the speed of the camera itself .Changing the optics of the camera (using a lens with higher magnification) and using a faster camera should allow to improve the results.

\section{Conclusions}

The aim the research presented in this article was to broaden the understanding of the liquid transport in textiles, as the knowledge of the transport phenomena allows for the design of textiles for various applications, such as comfortable to wear wound dressings. To visualise liquid transport through textiles, three test methods were used: NMR/MRI, thermography and high speed video imaging. All three chosen methods proved to be suitable for testing of the liquid transport and distribution in textiles, but each one of them was best suited for analysing specific types of samples due to their limitations. The best methods for testing thick and multilayered fabrics were NMR/ MRI and the high-speed video camera, whereas for thinly woven fabrics a thermographic camera with adapted optics proved to be the best tool.

The tests were carried on two new types of textiles: a duellayer knitted fabric and a woven fabric, both made from hydrophilic and hydrophobic fibres (cotton, viscose and polypropylene). The knitted fabrics were tested as a new type of wound dressing, which transported moisture with adequate control; therefore, it could be used as a substrate for a drug delivery system. Moisture transport within textiles was enabled by the pores in the fabric structure. In the doublelayered knitted fabrics, moisture was transported between fibres in the hydrophobic layer, and next chemically bonded in hydrophilic layer. The dynamics of moisture transport proved to be dependent on the type of material used to create the textile and its structure.

\section{Acknowledgements}

The research work was financed by the European Union under the VI Frame Program as a project titled "Multifunctional Medical Textiles for Wound (e.g., Decubitus) Prevention and Improved Wound Healing", Contract No. NMP2-CT-2006-026741.

The authors would like to thank Mr. Krzysztof Kaczorek for his input into the design and for fabricating of the sample holder used for the NMR experiment.
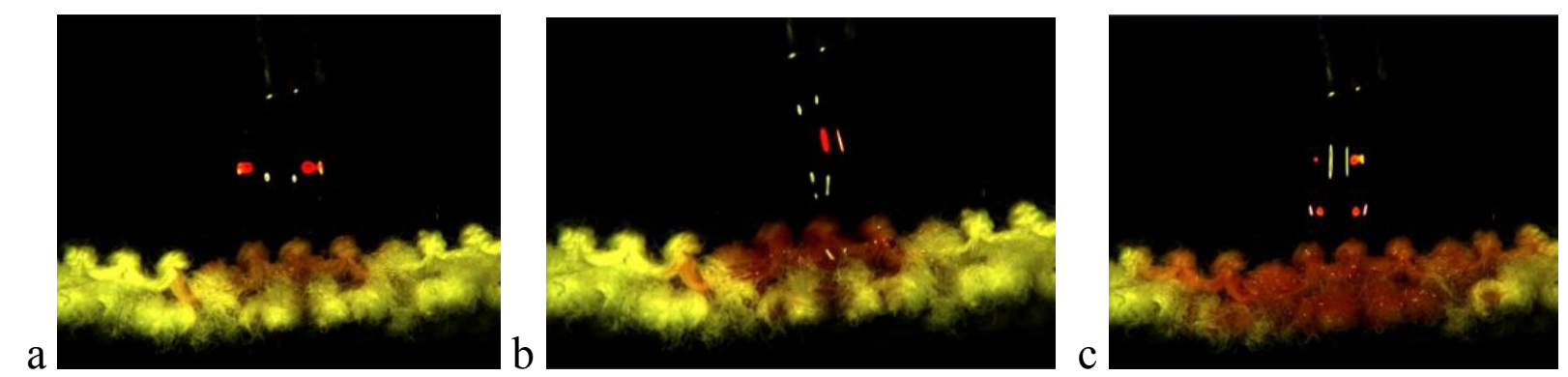

Figure 16. Moisture flow within knitted fabric. a - initial state, b - channels filling with liquid in the hydrophilic layer, c -saturated hydrophilic layer.

a

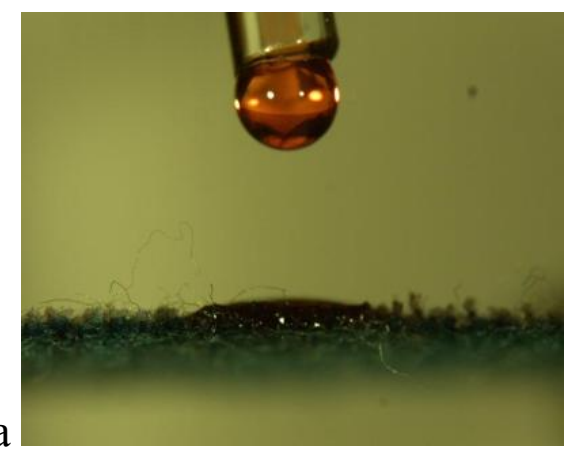

b

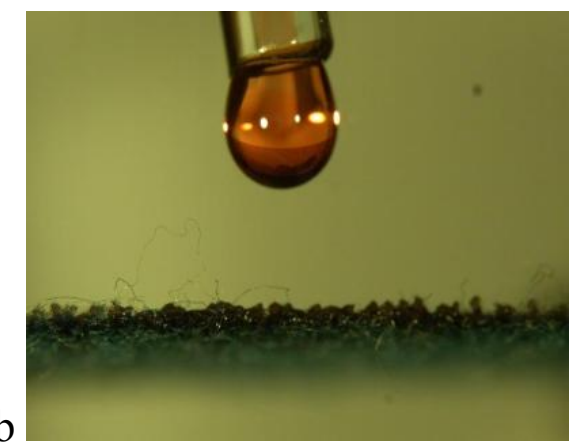

Figure 17. Moisture flow within the woven fabric. a -wetting of the sample, $b$ - the woven fabric with absorbed liquid. 


\section{References}

[1] Thermal and moisture transport in fibrous materials, ed. N. Pan, University of California and P. Gibson, US Army Soldier Systems Center, USA, Woodhead Textiles Series, No. 56.

[2] R.H. Dettre, R.E. Johson (1964) in "Contact Angle, Wettability and adhesion" (ed. R. F. Gould), Advances in Chemistry Series, Vol.43. ACS, Washington, D.C, p. 136.

[3] E.M. Sanders, S.H. Zeronian (1982). J. App. Poly. Scie., 27 (11), 4477-4491.

[4] N. Özdil, G. Süpüren, G. Özçelik, J. Průchová, „A study on the moisture transport properties of the cotton knitted fabrics in single jersey structure".

[5] G. Song, Numerical simulations of heat and moisture transport in thermal protective clothing under Flash Fire
Condition. International Journal of Occupational Safety and Ergonomics (JOSE), 2008, vol. 14, no. 1, pp. 89106.

[6] R.L. Barker, H. Hamouda, A. Kuznetsov, D.B. Thompson, Modelling heat and moisture transport in fire fighter protective clothing during flash fire exposure, National Textile Central Research Briefs. Management systems competency: June 2003.

[7] Vincent Maida, Marguerite Ennis, Craig Kuziemsky, and Linda Trozzolo, RN Symptoms Associated with Malignant Wounds: A Prospective Case Series, Journal of Pain and Symptom Management Vol. 37, No. 2, February 2009.

[8] Yi Luo, Kelly R. Kirker, Glenn D. Prestwich Cross-linked hyaluronic acid hydrogel films: new biomaterials for drug delivery, Journal of Controlled Release, vol. 69 (2000), pp. 169-184. 